\title{
The neurohormone orexin stimulates hypoxia-inducible factor-1 activity
}

\author{
Devanjan Sikder and Thomas Kodadek ${ }^{1}$ \\ Division of Translational Research, Departments of Internal Medicine and Molecular Biology, University of Texas \\ Southwestern Medical Center, Dallas, Texas 75390-9185, USA
}

\begin{abstract}
Orexin A and Orexin B (also known as hypocretins) are neuropeptides that bind two related G-coupled protein receptors (OXR1 and OXR2) and thus induce wakefulness, food consumption, and locomotion. Conversely, deletion of the orexin gene in mice produces a condition similar to canine and human narcolepsy. Despite the central importance of the orexin system in regulating wakefulness and feeding behavior, little is known about the downstream signaling mechanisms that achieve these effects. In this study, genomics techniques are used to probe this question and reveal that orexin activates the hypoxia-inducible factor 1 (HIF-1), a heterodimeric transcription factor whose pathogenic role in stimulating angiogenesis in hypoxic tumors has been the focus of intense investigation. Orexin-stimulated HIF-1 activity is due to both increased HIF-1 $\alpha$ gene transcription and a down-regulation of von Hippel-Lindau (VHL), the E3 ubiquitin ligase that mediates the turnover of HIF-1 via the ubiquitin-proteasome pathway. Orexin-mediated activation of HIF-1 results in increased glucose uptake and higher glycolytic activity, as expected from studies of hypoxic cells. However, orexin receptor-expressing cells somehow override the HIF-1-mediated preference for funneling pyruvate into anaerobic glycolysis and instead favor ATP production through the tricarboxylic acid cycle and oxidative phosphorylation. These findings implicate HIF-1 as an important transcription factor in the hormone-mediated regulation of hunger and wakefulness.
\end{abstract}

[Keywords: Hunger; hypoxia-inducible factor; orexin; signaling; sleep]

Supplemental material is available at http://www.genesdev.org.

Received June 18, 2007 revised version accepted September 24, 2008.

Mammalian cells depend on receptor signaling to regulate the uptake of metabolic fuels. The hypothalamus is largely responsible for mediating these vital adaptations by sensing and coordinating orchestrated changes in wakefulness, appetite, and metabolism. Orexin A and Orexin B are closely related hypothalamic neuropeptides that are intimately involved in this regulatory network (Hungs and Mignot 2001; Willie et al. 2001). The orexins mediate their effects by binding to two G-proteincoupled receptors, OXR1 and OXR2. Mice lacking orexins or the cognate receptors display striking phenotypes. They eat less compared with matched littermates and, perhaps most interestingly, display a sleep disorder that is remarkably similar to human (Chemeilli et al. 1999) and canine (Lin et al. 1999) narcolepsy. Indeed, while human narcoleptics generally do not carry mutations in the orexin gene, they are deficient in orexin-producing neurons, making narcolepsy one of the few sleep disorders with a well-defined molecular cause (Scammell 2001). However, despite the high level of interest in better understanding sleep and energy homeostasis, little is

${ }^{1}$ Corresponding author.

E-MAIL thomas.kodadek@utsouthwestern.edu; FAX (214) 648-1450.

Article is online at http://www.genesdev.org/cgi/doi/10.1101/gad.1584307. known about the molecular pathways by which the orexins and their cognate receptors function. In this report, we begin to address this question using global gene expression analysis. Of the many orexin-responsive genes identified, we found that the hypoxia-inducible factor-1 (HIF-1) and many of its target genes are induced strongly. More directed experiments confirmed that this also applies to hypothalamic neurons.

The heterodimeric HIF-1 transcription factor has been studied intensively as a key component in the response to hypoxia (Kaelin 2005). Under normoxic conditions, one of the two subunits, HIF-1 $\alpha$, is hydroxylated by iron and oxygen-dependent enzymes at specific proline residues (Bruick and McKnight 2001; Epstein et al. 2001). These post-translational modifications result in its recognition by an E3 ubiquitin ligase complex that includes the von Hippel-Lindau (VHL) protein, which subsequently mediates it destruction via the ubiquitin-proteasome pathway (Conaway and Conaway 2002). When the oxygen concentration is low, HIF- $1 \alpha$ is hydroxylated less efficiently and the protein accumulates, triggering the transcription of its target genes, which include, among others, genes that encode angiogenic (Hirota and Semenza 2006) and glycolytic (Semenza et al. 1994) proteins that are crucial for cell survival and growth under 
these conditions. This pathway is of central interest in the biology of hypoxic solid tumors and other cancers. However, HIF-1 has not been implicated as a central player in the regulation of feeding behavior or sleep/ wake cycles. We show here that orexin-mediated HIF-1 induction proceeds by a different mechanism that involves both induction of HIF- $1 \alpha$ gene expression as well as a reduction in VHL protein. We also demonstrate that the spectrum of HIF-1-induced genes is different in normoxic cells stimulated with orexin from that observed under hypoxia, particularly with respect to regulation of a critical metabolic branchpoint that determines the relative contribution of anaerobic glycolysis and oxidative phosphorylation in the production of ATP from glucose. A model is presented that attempts to place these results in an appropriate physiological context.

\section{Results \\ Identification of orexin-responsive genes through global expression profiling}

In order to probe the molecular events triggered by orexin binding to its receptor, we exposed stably transfected HEK293 cells expressing the orexin 1 receptor (OXR1) to either $100 \mathrm{nM}$ Orexin A peptide or the vehicle alone (5\% DMSO) for either 2 or $4 \mathrm{~h}$. The changes brought about in gene expression were then probed by global profiling using an Affymetrix HG-U133 plus two human genome arrays (Affymetrix). The transcriptional response is documented in Supplementary Table 1 (the MIAME description is provided under ArrayExpress accession: E-TABM-268). Two-hundred-sixty genes were found to be up-regulated and 64 were found to be downregulated by twofold or more in both the 2- and 4-h data sets (Supplementary Fig. 1). Consistent with previous observations, (Date et al. 1999; Yamanaka et al. 2000) the transcript levels for Fos (an indicator of neuronal activity and wakefulness) and neuropeptide $\mathrm{Y}$ (a potent stimulator of feeding behavior) were up modestly (Supplementary Table 1). Many of the more highly regulated transcripts were measured by quantitative RT-PCR analysis (Fig. 1A) and these data were in general agreement with that provided by the array analysis. Among the transcripts that were up-regulated were genes that encoded transcription factors, repressors and corepressors, GPCRs, GTPases, endocytosis machinery ion channels, proteins involved in cell cycle and cell proliferation, apoptosis, signal transduction, and metabolism (Supplementary Fig. 2). More than half of the responsive genes fell into two categories: cell growth $(30 \%)$ and metabolism $(27 \%)$ (Supplementary Fig. 2). Of the transcripts that were down-regulated, about a quarter are uncharacterized. The second largest category is comprised of genes involved in metabolism (17\%), followed closely by genes that play a role in cell-cell communication (Supplementary Fig. 2). These results suggest that orexin signaling has a profound impact on cellular metabolism.
A

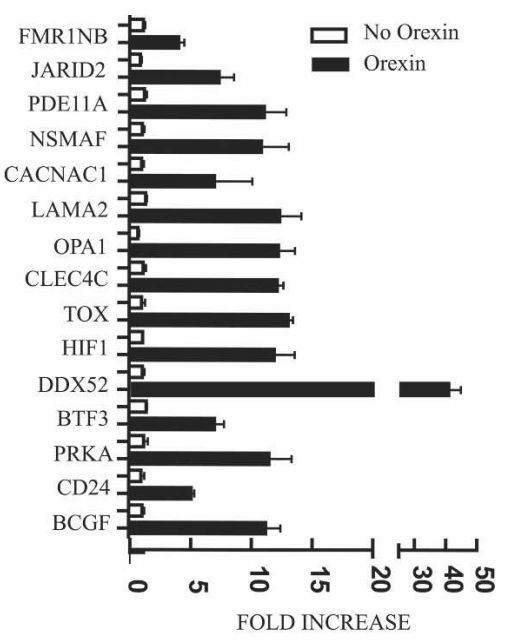

B

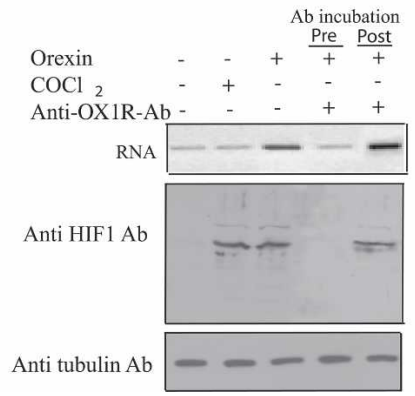

Figure 1. Validation of array-based study and quantitation of HIF- $1 \alpha$ level in orexin-treated cells. (A) HEK293 cells that stably express OXR1 were either treated with vehicle (5\% DMSO) or $100 \mathrm{nM}$ Orexin A peptide for $2 \mathrm{~h}$. RNA was isolated, converted to cDNA, and amplified by quantitative PCR using gene-specific primers. $(B)$ Orexin enhances HIF- $1 \alpha$ mRNA and protein levels. OXR1-expressing HEK293 cells were either treated with $5 \%$ DMSO (lane 1), $150 \mu \mathrm{M} \mathrm{CoCl}_{2}$ (simulating hypoxia) for $6 \mathrm{~h}$ (lane2), or $100 \mathrm{nM}$ orexin peptide in DMSO for $2 \mathrm{~h}$ (lane 3). Cells were preincubated with anti-OXR1 antibody $(\mathrm{Ab})$ for $1 \mathrm{~h}$, prior to treatment with orexin (lane 4) or treated with orexin for $2 \mathrm{~h}$ prior to incubation with anti-OXR1 antibody (lane 5).

\section{Orexin-mediated induction of HIF-1 $\alpha$}

The data were mined for functional patterns of altered gene expression through pathway analysis (Ingenuity). This "gene ontology"-based statistical analysis identified 16 networks that were above the threshold value. Cell survival and cell proliferation were most significant, which is depicted in Supplementary Figure 3. Among the canonical pathways identified by this analysis, TGF- $\beta /$ Smad/BMP, FGF, NF-кB, and hypoxic signaling were the most prominent. We decided to focus initially on the hypoxia pathway. HIF- $1 \alpha$, one of two components of the heterodimeric transactivator that mediates the hypoxic response, was the transactivator whose message was most highly up-regulated by orexin treatment (up to 15-fold) (Fig. 1A). The increase in HIF$1 \alpha$ transcripts is due to stimulation of transcription and not due to stabilization of mRNA message (Supplemen- 
tary Fig. 4). As mentioned above, HIF-1 has been of keen interest to the cancer biology community due to its critical role in stimulation of angiogenesis in oxygen-starved solid tumors (Kaelin 2005). While the normal physiological roles and regulation of HIF-1 are much less well understood, this protein has been linked to homeostatic regulation of metabolism (Simon 2006), a function common to orexins.

To determine whether this large increase in HIF- $1 \alpha$ transcript results in a similar increase in the HIF- $1 \alpha$ protein, Western blots using an anti-HIF-1 $\alpha$ antibody were performed. As shown in Figure 1B, extracts prepared from cells not treated with orexin exhibited little or no detectable HIF-1 $\alpha$, whereas treatment with orexin resulted in a large increase in protein level. This orexinmediated induction of HIF-1 $\alpha$ protein could be blocked by incubation of the cells with an anti-OxR1 antibody prior to treatment with orexin, but not subsequent to treatment with the hormone (Fig. 1B), confirming that this effect is due to signaling triggered by orexin-orexin receptor binding.

To determine whether the induced protein was active, the cells were transfected with a HIF-1-responsive reporter gene. As shown in Figure 2A, treatment of these cells under normoxic conditions resulted in a large increase in reporter gene expression that was dependent on the presence of HIF-1-binding sites in the promoter. Under hypoxic conditions, treatment of the cells with orexin resulted in little or no additional stimulation of the already high levels of reporter gene expression. This indicates that the orexin-mediated and hypoxia-mediated pathways for activation of HIF-1 activity do not syn-
A

B
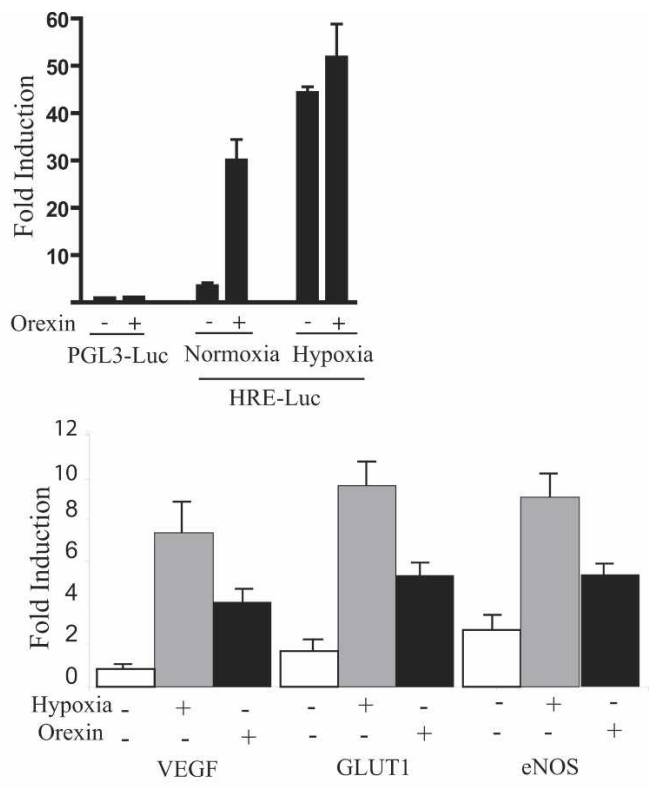

$\mathrm{C}$

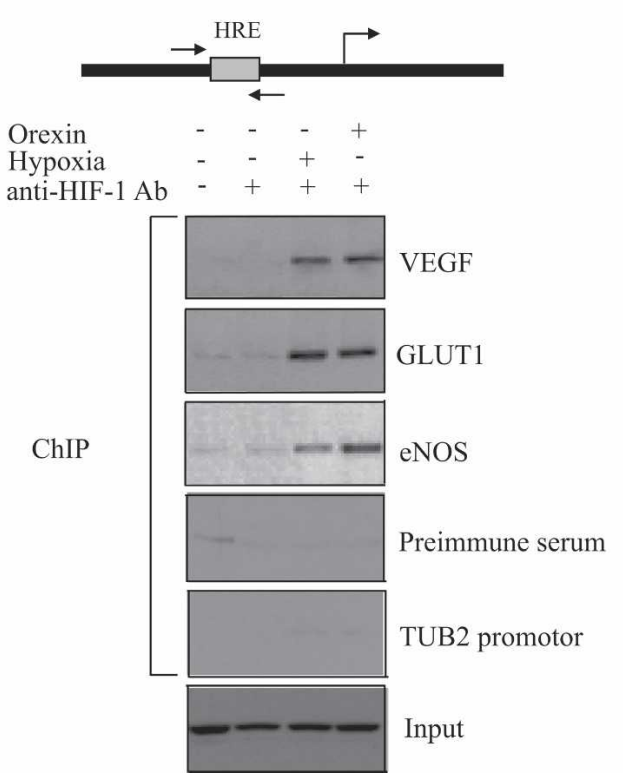

Figure 2. HIF-1 is transcriptionally active in orexinstimulated cells. All assays were conducted in HEK293 cells that stably express OXR1. (A) Reporter gene assay showing the response of a Luciferase reporter gene containing three HIF-1 response elements (HREs) in the promoter to orexin under normoxic and hypoxic conditions. PGL3-Luc is a control plasmid lacking the HREs. The experimental values are normalized with respect to a Firefly/Renilla luciferase control (empty vector-Luc). (B) Quantitative RT-PCR-based measurement of the induction of VEGF, GLUT1, and eNOS transcription in response to hypoxia and orexin. Ct values of target transcripts are referenced to that of $\beta$-tubulin in untreated samples and analyzed by $\Delta \Delta \mathrm{CT}$ method (Livak and Scmittgen 2001). (C) ChIP assays using antibodies against HIF-1 and primers specific to the promoters indicated. 
ergize. Orexin treatment also resulted in the stimulation of transcription of the endogenous VEGF, GLUT1, VEGFR3, eNOS genes, all of which are known to be regulated by HIF-1 (Fig. 2B), although the level of induction was somewhat weaker than that brought about by hypoxia. Chromatin immunoprecipitation (ChIP) assays (Fig. 2C) revealed strong stimulation of HIF-1 occupancy of the promoters of these genes when the cells were treated with orexin or subjected to hypoxic conditions. We therefore conclude that the orexin-induced HIF-1 complex is transcriptionally active.

The data shown in Figures 1 and 2 were obtained in an embryonic kidney cell line stably expressing OXR1. To

Figure 3. Orexin signaling induces Hif- $1 \alpha$-mediated transcription in hypothalamic neurons. Hypothalamic explants were prepared from the brains of wild-type mice or animals lacking OxR1 gene and cultured in DMEM in the presence of vehicle (5\% DMSO) or exposed to hypoxia ( $1 \%$ oxygen for $6 \mathrm{~h}$ ) or OrexinA peptide (100 $\mathrm{nM}$ for $2 \mathrm{~h}$ ). Slices were then processed for Western blots using antibodies raised against Hif- $1 \alpha$ and Hif-1 $\beta(A)$ and ChIP assays (in the wild-type neurons) using antibodies raised against HIF- $1 \alpha$ and gene-specific primers complementary to the indicated promoters $(B)$. The Ct values for each promoter in the experimental samples are referenced to that of untreated samples. Quantitative RT-PCR showing the response of the genes indicated to hypoxia and orexin in wild-type neurons $(C)$, and comparing the response of the indicated genes to orexin in wild-type and OXR1 knockout neurons $(D)$. Ct values of target transcripts are referenced to that of $\beta$-tubulin in untreated samples and analyzed by $\Delta \Delta \mathrm{CT}$ method (Livak and Scmittgen 2001)

A

B

C

D determine whether orexin-mediated induction of HIF- $1 \alpha$ levels and HIF-1 activity is of physiological significance, hypothalamic slices were prepared from eight wild-type mice and cultured. As shown in Figure 3A, treatment of these neurons results in a strong induction in HIF- $1 \alpha$ protein level, as determined by Western blotting. To assess HIF-1 activity in these cells, the hypothalamic slices were either left untreated, subjected to hypoxia for $6 \mathrm{~h}$, or treated with orexin for $2 \mathrm{~h}$, and the levels of several HIF-1-regulated transcripts were measured by quantitative RT-PCR. The results show that both hypoxia and orexin treatment of the neurons resulted in a four- to eightfold induction of these genes (Fig. 3B). The slices
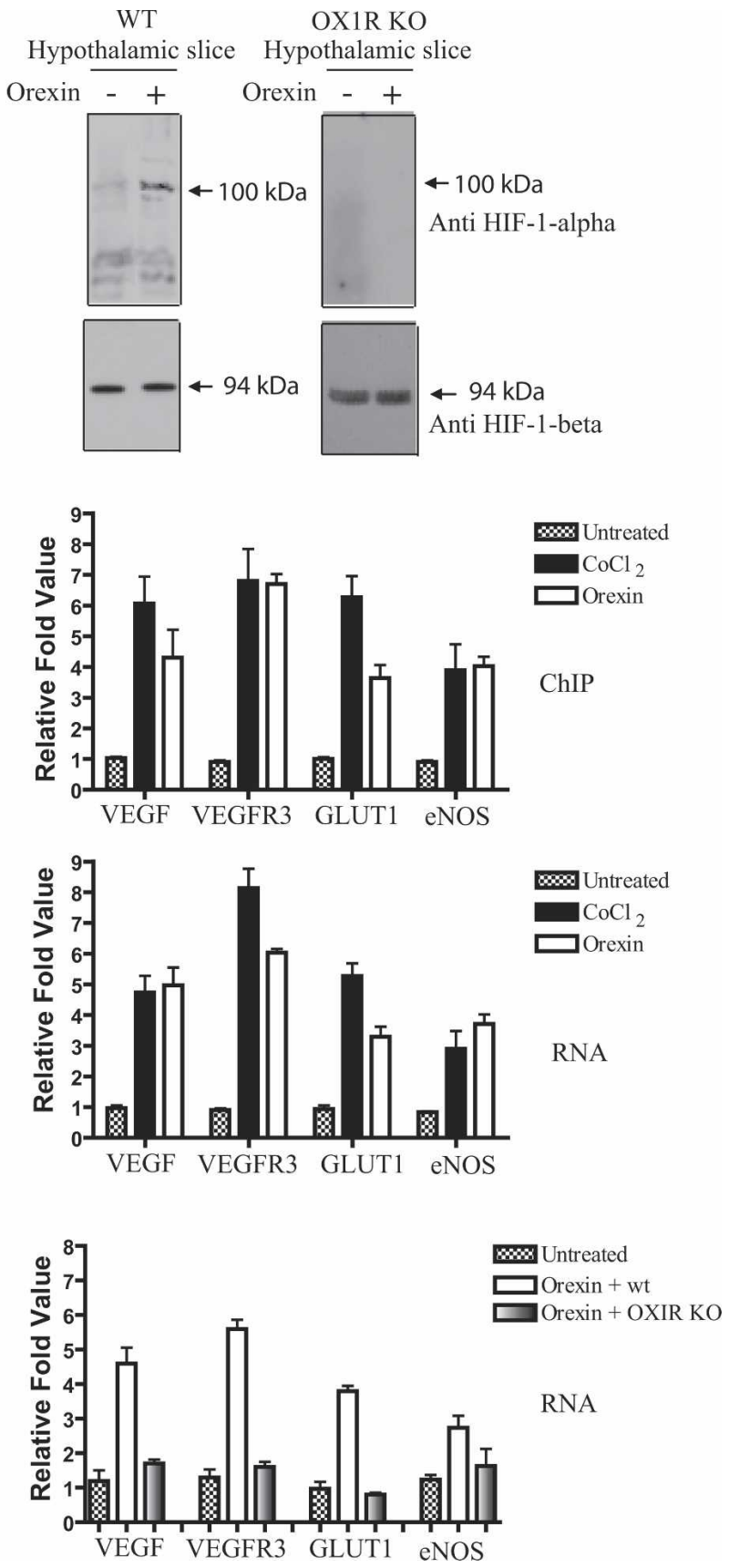
were then processed for ChIP analysis using an anti-HIF$1 \alpha$ antibody. As anticipated from the RNA levels, strong stimulation of HIF-1 association with the promoters of these genes was induced by hypoxia as well as orexin treatment (Fig. 3C). When the expression analysis was repeated with hypothalamic slices prepared from OXR1 knockout (KO) mice, the effect of hypoxia was identical (data not shown), but addition of orexin failed to stimulate expression of the genes (Fig. 3D). In addition, HIF-1 $\alpha$ levels were not increased substantially by orexin treatment (Fig. 3A, right panel). This confirms that the increase in HIF- $1 \alpha$ protein and subsequent activation of its target genes is triggered by orexin binding to its cognate receptor.

\section{A bipartite mechanism for orexin-mediated HIF-1 $\alpha$ induction}

In hypoxic cells, HIF-1 $\alpha$ levels rise due to less-efficient hydroxylation of a key proline residue on the protein. This post-translational modification is required for efficient recognition of HIF-1 $\alpha$ by the VHL-containing E3 ubiquitin ligase complex that triggers the proteasomemediated degradation of the protein (Kaelin 2005). Since the effect of orexin is observed under normoxic conditions, we speculated that the mechanistic basis of the rise in HIF-1 $\alpha$ level might be different. The genomic profiling data obtained in the HEK293 cells provided a clue, in that VHL gene expression was down-regulated upon treatment with orexin. To verify this result, quantitative RT-PCR and Western blotting were used. Figure 4A con-

A
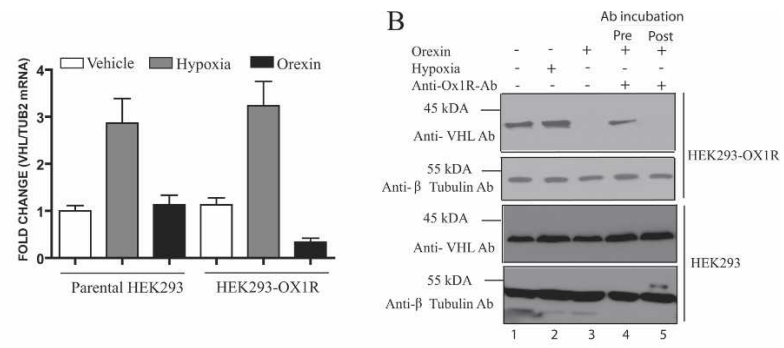

C

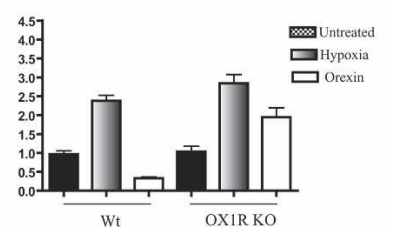

D

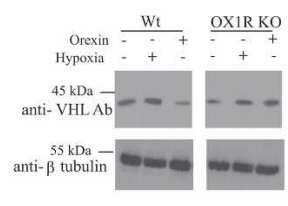

Figure 4. Effect of orexin on VHL levels in OXR1-expressing HEK293 cells and hypothalamic neurons. The effects of orexin treatment and hypoxia on VHL mRNA and protein levels were measured by quantitative RT-PCR and Western blotting, respectively, in OXR1-expressing HEK293 cells (A shows RNA levels and $B$ shows protein levels) or hypothalamic slices prepared from wild-type or OXR1 knockout mice $(C$ shows RNA levels and $D$ shows protein levels). In $A$ and $B$, anti-OXR1recepter $\mathrm{Ab}$ was incubated prior or post-orexin treatment. firms a reduction in VHL mRNA levels and the Western blot in Figure 4B demonstrates a dramatic orexininduced drop in VHL protein level in the cell culture model. Orexin did not alter the levels of either VHL mRNA or protein in the parental HEK293 cells. Blocking OX1R with $\mathrm{Ab}$ prior to orexin treatment significantly dampens this response.

To determine whether this was of physiological significance, the same experiment was repeated in cells cultured from hypothalamic slices prepared for wild-type and OXR1 knockout mice. As shown in Figure 4, C and $\mathrm{D}$, treatment of the cells derived from wild-type mice resulted in a reduction of VHL mRNA and protein levels of three- to fourfold, whereas orexin treatment had little effect on VHL gene expression and protein level in the cells lacking the orexin receptor. While the effect was less dramatic at the protein level than that observed in the embryonic kidney cell culture model, these results, combined with the data shown in Figure 1A, argue that the mechanism of orexin-induced stimulation of HIF- $1 \alpha$ levels is bipartite. The hormone-induced signaling cascade both up-regulates the level of HIF-1 $\alpha$ gene transcript and decreases the rate of protein degradation through reduction of VHL protein. We note that up-regulation of HIF-1 $\alpha$ levels under normoxic conditions has also been observed in stromal cells of hemangioblastomas lacking VHL function (Maxwell et al. 1999).

\section{Orexin increases glucose utilization and ATP production through HIF-1}

These data show clearly that orexin binding to its cognate receptor activates the HIF-1 transcription factor in the hypothalamus and implicates HIF-1 as an important player in the molecular mechanisms that modulate wakefulness and feeding behavior. What does this mean metabolically? In considering this point, it is important to remember that orexinergic neurons of the lateral hypothalamus act as glucose sensors. When glucose levels are low, orexin production is enhanced and visa versa (Yamanaka et al. 2003; Burdakov et al. 2006). This makes physiological sense, since orexin promotes wakefulness, hunger, and locomotion, exactly the state an organism should be in if blood sugar levels are low (i.e., the organism must search for a meal). Under hypoxic conditions, HIF-1 stimulates glycolysis and glucose utilization, among other metabolic effects (vide infra), in order to allow cells to meet their energy needs. Does HIF-1 stimulate glucose utilization when triggered by orexin?

The global analysis of gene expression reported here reveals the induction of many genes involved in energy homeostasis, including the facultative glucose transporters GLUT1 and GLUT3 and glycolytic genes (Figs. 2, 3; Supplementary Table 2). Many of these genes are known to be HIF-1 responsive (Semenza et al. 1994; Baumann et al. 2007). Does this increased level of the glucose transporter mRNAs indeed result in increased sugar utilization upon orexin stimulation? To address this point, cells were cultured overnight in DMEM and then treated with orexin. Aliquots of the conditioned medium were 
assayed for glucose utilization. As seen in Figure 5A, orexin treatment significantly up-regulated glucose uptake from the medium when assayed both 2 and $4 \mathrm{~h}$ after hormone treatment. To determine whether this orexintriggered effect was manifest through the activity of HIF- $1 \alpha$, we used small interfering RNA (siRNA) to knock down HIF-1 $\alpha$ expression by at least 90\% (Supplementary Fig. 5) and then assayed for orexin-induced glucose utilization. As shown in Figure 5A, no significant hormone-dependent increase in glucose utilization was observed in the HIF- $1 \alpha$-deficient cells. This demonstrates that orexin-stimulated glucose utilization is mediated through HIF- $1 \alpha$.

To determine whether the orexin-mediated increase in glucose utilization resulted in increased cellular energy production, we measured cellular ATP levels in the presence or absence of the hormone. OXR1-expressing cells were cultured overnight and treated with orexin. ATP measurements were normalized to total cellular protein as described (see Materials and Methods). As seen in Fig-

A

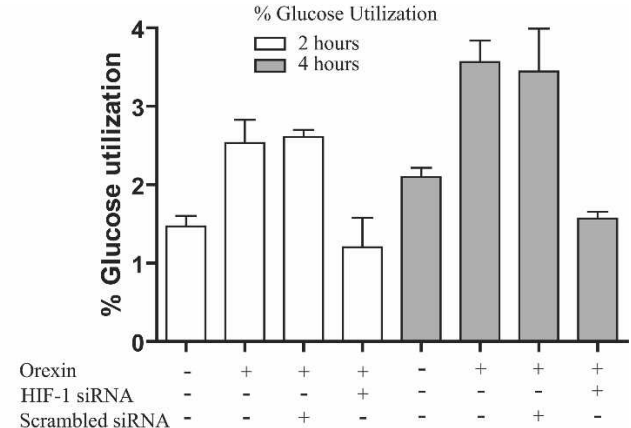

B

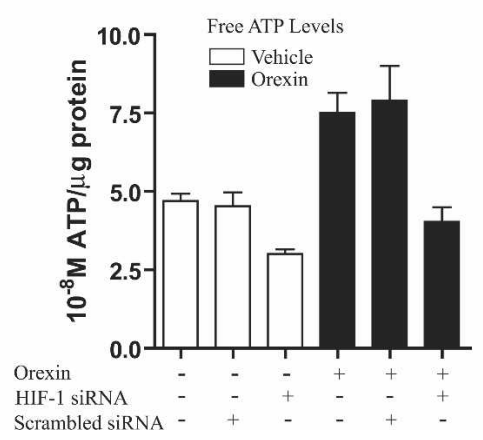

Figure 5. Orexin stimulates glucose utilization and cellular ATP levels. (A) OXR1-expressing cells cultured overnight in DMEM that had been treated with a HIF- $1 \alpha$-specific siRNA or a control siRNA were treated either with vehicle or with $100 \mathrm{nM}$ orexin. An aliquot of the medium was taken out before and after treatment at the indicated time points and assayed for glucose levels. The amount of glucose depleted after $2 \mathrm{~h}$ and $4 \mathrm{~h}$ were divided by the respective values at the zero time point and expressed as percent glucose utilization. $(B)$. Cultured cells that had been treated with a HIF- $1 \alpha$-specific siRNA or a control siRNA were treated either with vehicle or with $100 \mathrm{nM}$ orexin for $2 \mathrm{~h}$ and ATP measurements were performed. Signals were normalized to total cellular protein present in the same cell extract. ure 5B, total cellular ATP content increased significantly in orexin-treated cells compared with untreated cells. To assess the role of HIF-1 in this process, we again severely reduced the level of HIF- $1 \alpha$ using siRNA and repeated the experiment. As shown in Figure 5B, knockdown of HIF- $1 \alpha$ levels abrogated the effect of orexin treatment on ATP levels observed in untreated cells. A similar response was seen when ATP levels were measured $4 \mathrm{~h}$ after orexin treatment under the various conditions described above (data not shown). Taken together, these data argue strongly that HIF-1 is a key factor in mediating the orexin-dependent up-regulation of cellular energy metabolism.

\section{Orexin signaling shunts pyruvate preferentially} into the tricarboxylic acid (TCA) cycle independent of HIF-1 activity

The data described above suggest that a major physiological role of HIF-1 is to help to modulate cellular energy utilization in response to extracellular signaling (also see Lum et al. 2007). In this view, hypoxic tumors can be viewed as co-opting this activity of HIF-1 for pathophysiological purposes. However, there is a quandary in reconciling the data shown above with what has been gleaned from studies of hypoxic cells. Glycolysis generates pyruvate as an end product, which has two major fates. It can be reduced to lactate by $\mathrm{NADH}$ and lactate dehydrogenase (LDHA), thus facilitating anaerobic glycolysis, or can be converted irreversibly to Acetyl CoA by the pyruvate dehydrogenase (PDH) complex, priming the TCA cycle and oxidative phosphorylation in the mitochondria. Thus, the fate of pyruvate is an important metabolic branchpoint (Fig. 6A). In hypoxic cells, the pyruvate-to-lactate conversion is favored strongly, which makes sense since there is little oxygen to drive oxidative phosphorylation. HIF-1 plays a major role in controlling this branchpoint. LDHA is known to be a HIF-1 target gene (see above). Moreover, it has been found recently that $\mathrm{PDH}$ kinase (PDK1), a negative regulator of PDH activity, is also a HIF-1-regulated gene (Kim et al. 2006; Papandreou et al. 2006). Thus, in hypoxic cells, HIF-1 actively promotes LDHA function and suppresses PDH activity to shift metabolic flux from oxidative phosphorylation to anaerobic glycolysis (Simon 2006). However, it is not obvious why this would be desirable in normoxic neurons, since oxidative phosphorylation is a more efficient way to generate ATP from sugar. Thus, either the HIF-1-mediated transcription program is somewhat different in orexin-stimulated and hypoxic cells, or counter intuitively, orexin signaling forces cells to use mostly anaerobic glycolysis for energy production.

When we queried the global gene expression data set, it showed that orexin treatment of HEK293 cells results in an increase in PDH expression and decreased expression of both PDK1 and LDHA, a situation that would strongly favor shunting pyruvate through the TCA cycle. Since global gene expression data can sometimes be misleading, we first examined the levels of these three genes 

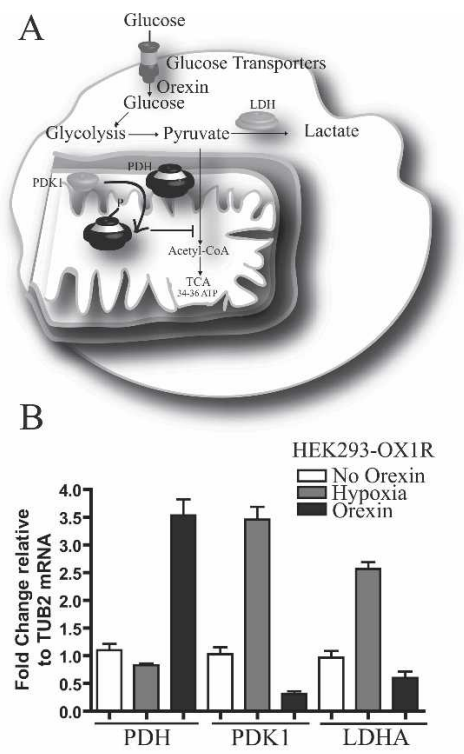

C

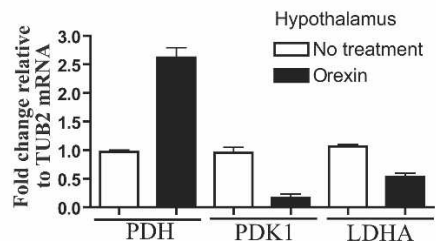

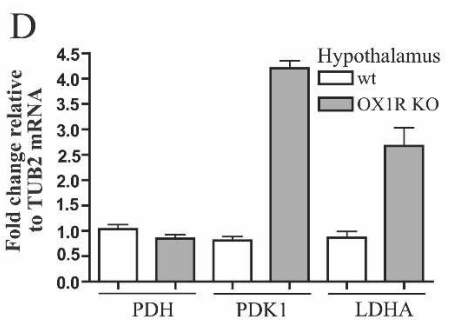

$\mathrm{E}$

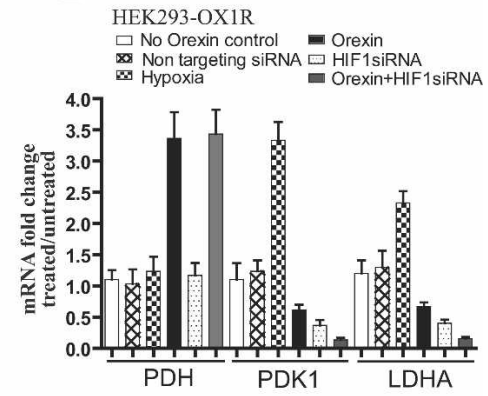

Figure 6. Effect of hypoxia and orexin on the expression of the LDHA, PDH, and PDK1 genes, which determines the fate of pyruvate. (A) Schematic illustrating the metabolic branchpoint determined by the fate of pyruvate, which is the end product of glycolysis. Reduction to lactate by LDHA and NADH facilitates anaerobic glycolysis, while irreversible conversion to acetyl-CoA by PDH fuels the TCA cycle and oxidative phosphorylation. PDK1 inhibits the activity of $\mathrm{PDH}$ through phosphorylation. (B) Effect of hypoxia and orexin treatment on the expression of LDHA, PDH, and PDK1 in HEK293 cells that stably express OXR1. (C) Effect of orexin (100 nM) on the expression of $\mathrm{PDH}$, PDK1, and LDHA in wild-type hypothalamic neurons. (D) Expression of PDH, PDK1, and LDHA in wild-type and OXR1 knockout hypothalamic neurons. These cells were not treated with exogenous orexin. (E) Effect of HIF-1 $\alpha$ knockdown on the levels of the indicated transcripts in OXR1-expressing HEK293 cells. by quantitative RT-PCR in orexin receptor-expressing HEK293 cells subjected to hypoxia or treated with orexin under normoxic conditions. Consistent with previous reports, hypoxia resulted in a stimulation of LDHA and PDK1 expression, with little or no effect on PDH message levels. In contrast, orexin treatment results in a modest down-regulation of LDHA and a larger decrease in PDK1 expression, as well as a 3.5-fold increase in PDH message levels (Fig. 6B). Clearly therefore, there is indeed a major difference in the expression of these HIF-1-regulated genes in hypoxic and normoxic, orexin-treated cells.

To determine whether this result is peculiar to the cell culture model or whether it represents physiologically meaningful regulation, the effect of orexin treatment on the expression of these genes in hypothalamic cells was examined. As shown in Figure 6C, the results were quite similar. Finally, we also isolated hypothalamic slices from the brains of wild-type and OXR1 knockout mice and measured the steady-state levels of these three messages in each. Note that in this case, neither the wildtype nor OXR1 knockout neurons were treated with orexin. As is shown in Figure 6D, the PDH message levels are approximately equal. The hypothalamic cells derived from the knockout mice have much higher levels of PDK1 and LDHA mRNA than the wild-type mice, consistent with the idea that the presence of the orexin receptor down-regulates the expression of these genes even without added exogenous orexin. A similar result was obtained when the whole-brain homogenate from these animals was examined (data not shown). To investigate whether these orexin-mediated changes were due to HIF-1 $\alpha$ activity, we knocked down HIF- $1 \alpha$ and measured the PDH, PDK1, and LDHA transcripts. The reduction of HIF-1 $\alpha$ did not block the orexin-mediated effect (Fig. 6E). It is noteworthy that the orexin-mediated decrease in PDK1 and LDHA gene expression is even more severe in cells where HIF-1 $\alpha$ was knocked down than in cells with normal HIF-1 $\alpha$ levels (Fig. 6E). This suggests that in orexin-stimulated cells HIF-1 does act to stimulate LDHA and PDK1 gene expression, but that this effect is overridden by some hormone-triggered inhibitory mechanism.

We next asked whether the differences seen in the transcription of PDH, PDK1, and LDHA in these cells resulted in the expected changes in metabolite levels. First, the amount of lactic acid produced by OXR1-expressing cells in response to orexin was determined and the role of HIF-1 in this process was addressed using siRNA knockdown of the $\alpha$ subunit. As shown in the Figure 7A, orexin inhibited the production of lactate at all of the time points studied, consistent with the transcription data (Fig. 6B). Knockdown of HIF1- $\alpha$ did not further inhibit the lactate production in the orexintreated cells. Interestingly, lactate production in the control cells not treated with orexin dropped substantially following HIF1- $\alpha$ knockdown. This suggests that a low level of HIF- $1 \alpha$ functions even in normoxic cells, consistent with the observation made by Lum et al. (2007). In any case, these data confirm that the orexin-mediated 


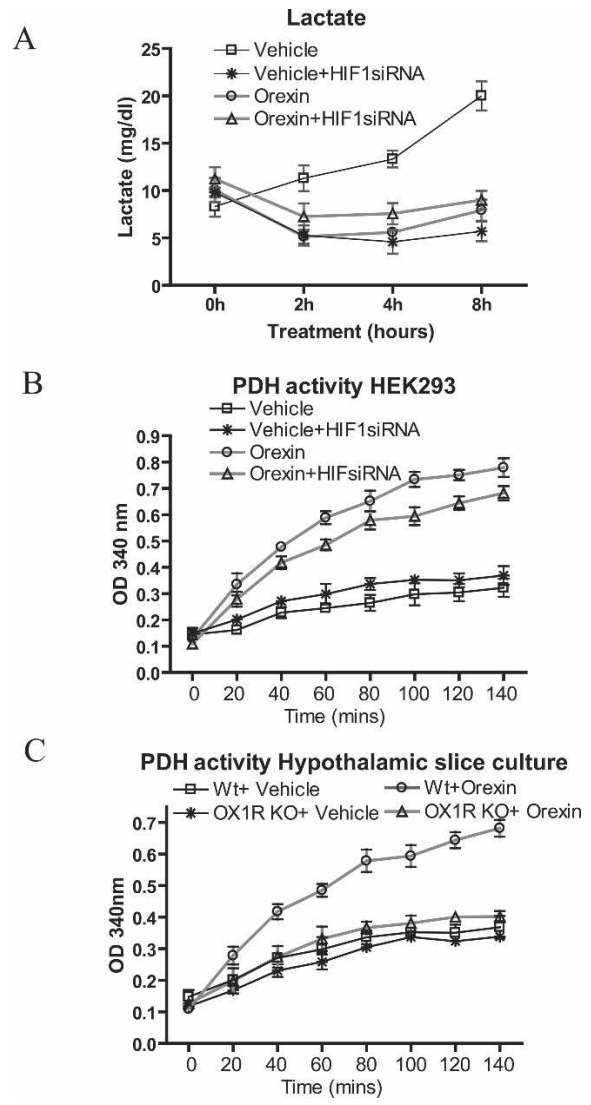

Figure 7. Roles of orexin and HIF-1 in regulating LDHA and PDH activity. (A) OXR1-expressing HEK293 cells treated with orexin or vehicle in the presence or absence of HIF-1 $\alpha$ or control siRNA. Lactate levels were measured at the times indicated. (B) OXR1-expressing HEK293 cells treated with orexin or vehicle in the presence or absence of HIF- $1 \alpha$ or control siRNA. $\mathrm{PDH}$ activity was measured at the time indicated. (C) $\mathrm{PDH}$ activity from hypothalamic explants of wild-type and OXR1null mice was assessed following immunocapture of $\mathrm{PDH}$.

decrease in LDHA gene expression indeed corresponds to a large drop in lactate production, as expected.

To determine whether orexin indeed promotes aerobic glycolysis as suggested by the transcription data (Fig. 6B), $\mathrm{PDH}$ was isolated from mitochondrial extracts of orexintreated and untreated cells, and its activity was measured. Orexin substantially stimulated $\mathrm{PDH}$ activity at all of the time points studied (Fig. 7B). A similar result was obtained in cells where the HIF-1 $\alpha$ level had been knocked down with siRNA (Fig. 7B). Orexin stimulated $\mathrm{PDH}$ activity in hypothalamic slice cultures prepared from mouse expressing OXR1. This response was absent in hypothalamic cultures prepared from OXR1-null mice (Fig. 7C).

These experiments confirm that both in the cell culture model and hypothalamic neurons, orexin signaling promotes metabolic flux through the TCA cycle and away from anaerobic glycolysis. They also show that the HIF-1 transcription factor, while clearly responsive to orexin at many other genes, does not play a significant role in mediating this metabolic branchpoint.

\section{Discussion}

We used a genomics approach to begin to deduce the signaling pathways used by orexin, an important regulator of hunger, feeding behavior, and sleep. Specifically, we expressed one of the orexin receptors in an endothelial cell line and used transcript profiling to identify genes whose expression was significantly up- or downregulated by treatment of the receptor-expressing cells with orexin. These data reveal an unanticipated connection between orexin signaling and HIF-1 activity (Figs. $1-3)$. We also demonstrated that the mechanism of HIF$1 \alpha$ accumulation is different from that observed in hypoxic cells. In addition to mediating a large increase in HIF-1 $\alpha$ transcript levels, orexin signaling resulted in a reduction in the level of VHL (Fig. 4), the E3 ubiquitin ligase that is responsible for triggering proteasomemediated turnover of this transactivator. This is distinct from the well-characterized mechanism for HIF-1 $\alpha$ buildup in hypoxic cells. VHL recognizes the prolinehydroxylated form of HIF- $1 \alpha$, a modification that is oxygen dependent. In hypoxia this modification is far less efficient, thereby sparing HIF-1 $\alpha$ from VHL-dependent proteasomal degradation. While these initial discoveries were made in a model cell line transfected with the orexin receptor, all of the major findings were reproduced in primary cultures of hypothalmic neurons. In addition, when such cultures were prepared from mice lacking the orexin receptor gene, treatment with the hormone did not stimulate HIF-1 activity. These results argue strongly for the physiological relevance of orexinmediated stimulation of HIF- $1 \alpha$ activity.

While hypoxia-induced HIF- $1 \alpha$ activity has been studied extensively with respect to the growth of oxygenlimited tumors, much less is known about the normal physiological roles of HIF-1 function. This study, along with a very recent report that HIF- $1 \alpha$ also responds to growth factor signaling (Lum et al. 2007), argues strongly that HIF-1 is a major physiological regulator of glucose metabolism.

The increased HIF- $1 \alpha$ activity resulted in a significant stimulation of glucose utilization and glycolytic activity (Fig. 5; Supplementary Table 2), as would be expected from studies of HIF-1 activity of hypoxic cells. However, a curious feature of our findings has to do with the fate of the pyruvate produced through increased glycolytic activity. Under hypoxic conditions, it is well documented that HIF-1 activity helps to favor pyruvate flux through anaerobic glycolysis (Fig. 6A). This is due, in part, to the fact that HIF-1 targets both the LDHA and PDK1 genes, stimulating their expression. LDHA processes pyruvate to lactate, whereas PDK1 phosphorylates $\mathrm{PDH}$, thus reducing its activity. However, measurement of both gene expression and metabolite levels showed that this is not the case in orexin-treated cells. They do make more ATP (Fig. 5B) and exhibit increased glucose uptake (Fig. 5A) and glycolytic activity. But measurements of the expression of LDHA, PDH, PDK1 (Fig. 6), lactate levels (Fig. 7A), and the activity of mitochondrial PDH (Fig. 7B,C) show clearly that orexin-stimulated cells preferentially 
shunt pyruvate into the TCA cycle and away from anaerobic glycolysis. Moreover, when these measurements were repeated in HIF- $1 \alpha$-depleted cells, the same was true (Figs. 6, 7), demonstrating that HIF-1 does not play a significant role in controlling this branchpoint in orexinstimulated cells.

Physiologically, this makes a great deal of sense. Orexin signals hunger when blood glucose levels are low, prompting an animal to search for food. In this state, it is obviously desirable to be alert, thus rationalizing the stimulation of "wakefulness" by the hormone. Based on the data shown here, one can hypothesize that perhaps part of what wakefulness means biochemically is an enhanced level of metabolism and energy production in neurons. Since processing of pyruvate through the TCA cycle and oxidative phosphorylation is by far the most efficient way to make ATP, one would imagine that normoxic cells responding to orexin stimulation would preferentially use this pathway, and this is the case. Also relevant to this point is a very recent report by Thompson and coworkers (Lum et al. 2007) that demonstrated HIF- $1 \alpha$ responds to growth factor signaling under normoxic conditions. In this case as well, HIF-1 up-regulates glycolysis and stimulates production of lactate from glucose. In other words, the transcription program is similar to that seen under hypoxic conditions, except in the latter case, the effects of HIF-1 are amplified greatly. Therefore, taking into account the data reported here and in previous reports of HIF-1 activity in growth factortreated and hypoxic cells, we can see that stimulation and glucose uptake and glycolysis appears to be a general function for the HIF-1 transcription factor that is important in both physiological and pathophysiological settings. However, the effect of HIF-1 on events downstream from pyruvate production is specific to the physiologic state of the cell. Given the current evidence, it appears likely that orexin receptor-expressing cells have operative in them some mechanism that overrides HIF-1-mediated activation of LDHA and PDK1, perhaps by inducing a repressor that targets these genes but not other HIF-1 target genes. This is consistent with the data shown in Figure 6, D and E, which show that even without being treated with orexin, neurons containing the orexin receptor exhibit lower levels of LDHA and PDK1 gene expression than cells isolated from an OXR1 knockout mouse (Fig. 6D). Treatment with orexin magnifies this effect (Fig. 6C) and this orexin-mediated diminution of LDHA and PDK1 expression is even more severe in cells where HIF-1 $\alpha$ levels were knocked down. Elucidating the detailed molecular mechanisms behind these events will be important in better understanding this regulatory network in the future.

\section{Materials and methods}

\section{Treatments}

Cells were cultured in DMEM with $1 \mathrm{mM}$ D-glucose supplemented with $10 \%$ FCS, $2 \mathrm{mM}$ glutamine, $100 \mathrm{U} / \mathrm{mL}$ penicillin, and $100 \mu \mathrm{g} / \mathrm{mL}$ streptomycin for $6 \mathrm{~h}$ in a humidified $37^{\circ} \mathrm{C}$ in- cubator at $94 \% \mathrm{~N}_{2}, 1 \% \mathrm{O}_{2}$, and $5 \% \mathrm{CO}_{2}$. When using $\mathrm{CoCl}_{2}$, HEK293 cells were exposed to $150 \mu \mathrm{M} \mathrm{CoCl}_{2}$ for $6 \mathrm{~h}$. Orexin A peptide (Sigma) was suspended in 5\% DMSO and was used at $100 \mathrm{nM}(\mathrm{Fc})$ for either 2 or $4 \mathrm{~h}$. For actinomycin D treatment, cells were incubated for $0-4.5 \mathrm{~h}(10 \mu \mathrm{g} / \mathrm{mL})$ following exposure to either $100 \mathrm{nM}$ orexin or vehicle. Cells were collected at the end of each time point and processed for assaying mRNA levels.

\section{Plasmid constructs}

pGL3-3XHOURE-Luc contained three copies of the HIF-1 response element (HRE) from the EPO promoter linked to thymidine kinase core promoter. pRL-TK (Promega) was used as a control for transfection efficiency.

\section{Antibodies}

Anti-HIF-1 $\alpha$ (NB 100-134) was from Novus Biologicals; antiVHL Ab (ab11189-250) and anti-LDHA Ab (AB53010) were purchased from Abcam; and anti-HIF-1- $\beta$ (sc-8077), anti-Ox1R (sc-8072), anti- $\beta$ tubulin (sc-5247), anti-GAPDH (sc-32233), and anti-PDK1 (sc-7140) antibodies were purchased from Santa Cruz Biotechnology, Inc.).

\section{Reporter gene assays}

OXR1-expressing HEK293 cells were plated in DMEM with 1 mM D-glucose supplemented with 10\% FCS, 2 mM glutamine, $100 \mathrm{U} / \mathrm{mL}$ penicillin, and $100 \mu \mathrm{g} / \mathrm{mL}$ streptomycin and were grown to $80 \%$ confluency. pGL3-HRE-Luc was transiently transfected into these cells using lipofectamine (Invitrogen). The cells were then treated with $100 \mathrm{nM}$ orexin for $2 \mathrm{~h}$. Cells were washed in PBS prior to assaying for dual luciferase assay. Activity of pGL3-HRE-Luc was normalized to the activity of pRL-TK transfection control and represented as relative luciferase activity on a bar graph.

\section{Hypothalamic explants}

Brains were dissected from decapitated OxR1 knockout mice and their wild-type littermates aged between 2 and 4 wk old in ice-cold PBS. The hypothalamus was isolated and 350- $\mu \mathrm{m}$-thick sections were prepared (Vibratome; Technical Products International) and placed in Krebs-Ringer bicarbonate buffer ( $\mathrm{pH} 7.4)$ for $1 \mathrm{~h}$ at $25^{\circ} \mathrm{C}$. These slices were placed on polycarbonate membranes and incubated in DMEM supplemented with 10\% FBS. Two hours later the medium was exchanged for serum-free medium and further incubated for $12 \mathrm{~h}$. Slice cultures were then either left untreated or treated with orexin or exposed to hypoxic conditions as described. For ChIPs, following the treatments, 350- $\mu \mathrm{m}$ slices were further minced, cross-linked in $1 \%$ formaldehyde for $15 \mathrm{~min}$ at room temperature, and processed as described (Tsankova et al. 2006).

\section{RNA isolation, RT-PCR, and Q-PCR analysis}

Total RNA was extracted from cells using Trizol reagent (Invitrogen) and converted to cDNA using a commercial reverse transcription system (Promega). Input cDNA was normalized and analyzed by the $\Delta \Delta \mathrm{CT}$ method and depicted on a bar graph (Livak and Scmittgen 2001). Q-PCR was performed using SYBR Green kit (Qiagen). PCRs were performed in i-Cycler (Bio-Rad). Actin RNA was used to normalize the input cDNA between samples. For ChIPs, primers corresponding to a noncoding sequence were used as internal control for normalization of sig- 
nals $\left(5^{\prime}\right.$-ATGGTTGCCACTGGGGATCT-3' ' 5' -TGCCAAAGC CTAGGGGAAGA-3').

\section{Whole-cell lysis and immunoblotting}

OXR1-expressing HEK293 cells were harvested by scraping and washing in ice-cold PBS and lysed in M-PER reagent (Pierce Chemical) supplemented with protease leupeptin $(5 \mu \mathrm{g} / \mathrm{mL})$, pepstatin $(1 \mu \mathrm{g} / \mathrm{mL})$, aprotinin $(2 \mu \mathrm{g} / \mathrm{mL})$, sodium fluoride $(50$ $\mathrm{mM})$, and sodium orthovandate $(2 \mathrm{mM})$. Following $10 \mathrm{~min}$ incubation on ice, lysates were centrifuged at $15,000 \mathrm{rpm}$ at $4^{\circ} \mathrm{C}$ and the supernatant was stored at $-80^{\circ} \mathrm{C}$. Protein estimation was performed using BCA assay (Sigma). Whole-cell lysates (25 $\mu \mathrm{g}$ of protein) were heat-denatured and resolved by $7.5 \%$ SDSPAGE under reducing conditions and then transferred to a nitrocellulose membrane. Blots were probed using antibodies against HIF- $1 \alpha$ (Upstate Biotechnology), HIF-1 $\beta$ (Santa Cruz Biotechnology), GAPDH, Tubulin2, VEGF, and eNos using the supplier's recommendation (Santa Cruz Biotechnology). AntiVEGFR3 antibody was a kind gift from Dr.Rolf Brekken (University of Texas Southwestern, Dallas, TX).

\section{Measurement of lactic acid}

Medium from cultured cells following $2 \mathrm{~h}$ of treatment with either vehicle or $100 \mathrm{nM}$ orexin were collected and lactic acid content was determined using a lactic acid assay kit (Sigma) according to the manufacturer's instructions.

\section{Measurement of glucose utilization}

Cells were seeded at 30,000 cells per well and cultured overnight in DMEM containing $25 \mathrm{mM}$ HEPES. A $50-\mu \mathrm{L}$ aliquot of the medium was taken out before cultured cells were treated (zero time point) either with the vehicle or $100 \mathrm{nM}$ orexin. Aliquots of $50 \mu \mathrm{L}$ of conditioned medium were taken out at 2and 4-h time point following treatment with the vehicle or 100 $\mathrm{nM}$ orexin. These aliquots were serially diluted and glucose content was measured using Amplex Red Glucose assay kit (Invitrogen). Background fluorescence (307 arbitrary units) was subtracted and the relative glucose in the medium was estimated from the standard curve generated. The amount of glucose depleted at the end of 2- and 4-h time points was divided by the respective values at the zero time point and expressed as percent glucose utilization.

\section{Measurement of free ATP}

Cells were seeded at 30,000 cells per well and cultured overnight in DMEM high-glucose medium containing $25 \mathrm{mM}$ HEPES. Cultured cells were treated either with vehicle or with $100 \mathrm{nM}$ orexin for 2 and $4 \mathrm{~h}$. ATP measurements were performed using CellTiter-Glo Assay kit (Promega) as recommended. Luminescence was measured using a Polarstar Optima microplate luminescence reader (BMG Labtech). Signals were normalized to total cellular protein present in the same cell extract using Bradford assay (Bio-Rad). The molar amount of ATP corresponding to the samples was estimated using a standard curve for ATP serially diluted in culture medium. Three independent experiments were performed with six replicates in each treatment group.

\section{HIF-1 $\alpha$ knockdown}

One-hundred microliters of cells $\left(2.5 \times 10^{5}\right.$ cells per milliliter $)$ were plated and cultured overnight at $37^{\circ} \mathrm{C}$ with $5 \% \mathrm{CO}_{2}$, in triplicate for each treatment, as recommended by the manufac- turer (Dharmacon). A scrambled nontargeting siRNA (Dharmacon) was used as a control. A transfection cocktail of siRNA (\#LQ-004018-00, Dharmacon) was complexed with DharmaFECT1 and delivered at a final concentration pf $100 \mathrm{nM}$ siRNA per well. Cells were cultured for $48 \mathrm{~h}$, at which point HIF- $1 \alpha$ mRNA was knocked down by $\sim 90 \%$ as estimated by quantitave RT-PCR. Cell viability was at least 93\% (Live/Dead Viability kit, Invitrogen).

\section{Measurement of PDH activity}

Cells $\left(2 \times 10^{7}\right)$ were transfected with or without HIF- $1 \alpha$-siRNA. After $48 \mathrm{~h}$, cultured cells were either treated with vehicle or $100 \mathrm{nM}$ orexin. Mitochondria was isolated as described (Ayllon et al. 2002). Fifty micrograms of mitochondrial lysate were used to immunocapture $\mathrm{PDH}$, and the associated activity was determined by assessing the reduction of $\mathrm{NAD}^{+}$to NADH at $340 \mathrm{~nm}$ using MitoProfile Assay kit (Mitosciences). To assess PDH activity from hypothalamic, explants were cultured and assayed as described above.

\section{Acknowledgments}

HEK293 cells stably transfected with the orexin receptor 1 were kindly provided by Professor Masashi Yanagisawa (University of Texas Southwestern). We also thank Professor Yanagisawa for several helpful discussions. Dr. Richard Bruick (University of Texas Southwestern) kindly provided pGL3-3XHOURE-Luc. This research was supported by the NHLBI Proteomics Initiative of the National Heart, Lung and Blood Institute, NIH, under contract number NO1-HV-28185.

\section{References}

Ayllon, V., Fleischer, A., Cayla, X., Garcia, A., and Rebollo, A. 2002. Segregation of Bad from lipid rafts is implicated in the induction of apoptosis. J. Immunol. 168: 3387-3393.

Baumann, M.U., Zamudio, S., and Illsley, N.P. 2007. Hypoxic upregulation of glucose transporters in BeWo choriocarcinoma cells is mediated by hypoxia-inducible factor-1 (HIF-1). Am. J. Physiol. Cell Physiol. 293: C477-C485. doi: 10.1152/ajpcell.00075.2007.

Bruick, R.K. and McKnight, S.L. 2001. A conserved family of prolyl-4-hydroxylases that modify HIF. Science 294: 13371340 .

Burdakov, D., Jensen, L.T., Alexopoulos, H., Williams, R.H., Fearon, I.M., O'Kelly, I., Gerasimenko, O., Fugger, L., and Verkhratsky, A. 2006. Tandem-pore $\mathrm{K}^{+}$channels mediate inhibition of orexin neurons by glucose. Neuron 50: 711-722.

Chemeilli, R., Willie, J.T., Sinton, C.M., Elmquist, J.K., Scammell, T., Lee, C., Richardson, J.A., Clay Williams, S., Xiong, Y., Kisanuki, Y., et al. 1999. Narcolepsy in orexin knockout mice: Molecular genetics of sleep regulation. Cell 98: 437451.

Conaway, R.C. and Conaway, J.W. 2002. The von HippelLindau tumor suppressor complex and regulation of hypoxia-inducible transcription. Adv. Cancer Res. 85: 1-12.

Date, Y., Ueta, Y., Yamashita, H., Yamaguchi, H., Matsukura, S., Kangawa, K., Sakurai, T., Yanagisawa, M., and Nakazato, M. 1999. Orexins, orexigenic hypothalamic peptides, interact with autonomic, neuroendocrine and neuroregulatory systems. Proc. Natl. Acad. Sci. 96: 748-753.

Epstein, A.C., Gleadle, J.M., McNeill, L.A., Hewitson, K.S., O'Rourke, J., Mole, D.R., Mukherji, M., Metzen, E., Wilson, M.I., Dhanda, A., et al. 2001. C. elegans EGL-9 and mam- 
malian homologs define a family of dioxygenases that regulate HIF by prolyl hydroxylation. Cell 107: 43-54.

Hirota, K. and Semenza, G.L. 2006. Regulation of angiogenesis by hypoxia-inducible factor 1. Crit. Rev. Oncol. Hematol. 59: $15-26$.

Hungs, M. and Mignot, E. 2001. Hypocretin/orexin, sleep and narcolepsy. Bioessays 23: 397-408.

Kaelin, W.G.J. 2005. The von Hippel-Lindau protein, HIF hydroxylation, and oxygen sensing. Biochem. Biophys. Res. Commun. 338: 627-638.

Kim, J.-w., Tchernyshyov, I., Semenza, G.L., and Dang, C.V. 2006. HIF-1-mediated expression of pyruvate dehydrogenase kinase: A metabolic switch required for cellular adaptation to hypoxia. Cell Metab. 3: 177-185.

Lin, L., Faraco, J., Li, R., Kadotani, H., Rogers, W., Lin, X., Qiu, X., de Jong, P.J., Nishino, S., and Mignot, E. 1999. The sleep disorder canine narcolepsy is caused by a mutation in the hypocretin (orexin) receptor 2 gene. Cell 98: 365-376.

Livak, K.J. and Scmittgen, T.D. 2001. Analysis of relative gene expression data using real-time quantitative PCR and the 2(- $\Delta \Delta \mathrm{C}(\mathrm{T}))$ Method. Methods 25: 402-408.

Lum, J.J., Bui, T., Gruber, M., Gordan, J.D., Deberardinis, R.J., Covello, K.L., Simon, M.C., and Thompson, C.B. 2007. The transcription factor HIF- $1 \alpha$ plays a critical role in the growth factor-dependent regulation of both aerobic and anaerobic glycolysis. Genes \& Dev. 21: 1037-1049.

Maxwell, P.H., Wiesener, M.S., Chang, G.W., Clifford, S.C., Vaux, E.C., Cockman, M.E., Wykoff, C.C., Pugh, C.W., Maher, E.R., and Ratcliffe, P.J. 1999. The tumour suppressor protein VHL targets hypoxia-inducible factors for oxygendependent proteolysis. Nature 399: 271-275.

Papandreou, I., Carins, R.A., Fontana, L., Lim, A.L., and Denko, N.C. 2006. HIF-1 mediates adaptation to hypoxia by actively downregulating mitochondrial oxygen consumption. Cell Metab. 3: 187-197.

Scammell, T.E. 2001. Wakefullness: An eye-opening perspective on orexin neurons. Curr. Biol. 11: R769-R771. doi: 10.1016/S0960-9822(01)00466-3.

Semenza, G.L., Roth, P.H., Fang, H.-M., and Wang, G.L. 1994. Transcriptional regulation of genes encoding glycolytic enzymes by hypoxia-inducible factor-1. J. Biol. Chem. 269: 23757-23763.

Simon, M.C. 2006. Coming up for air: HIF-1 and mitochondrial oxygen consumption. Cell Metab. 3: 150-151.

Tsankova, N.M., Berton, O., Renthal, W., Kumar, A., Neve, R.L., and Nestler, E.J. 2006. Sustained hippocampal chromatin regulation in a mouse model of depression and antidepressant action. Nat. Neurosci. 9: 519-525.

Willie, J.T., Chemelli, R.M., Sinton, C.M., and Yanagisawa, M. 2001. To eat or sleep? Orexin in the regulation of feeding and wakefullness. Annu. Rev. Neurosci. 24: 429-458.

Yamanaka, A., Kunii, K., Nambu, T., Tsujino, N., Sakai, A., Matsuzaki, I., Miwa, Y., Goto, K., and Sakurai, T. 2000. Orexin-induced food intake involves neuropeptide Y pathway. Brain Res. 859: 404-409.

Yamanaka, A., Beuckmann, C.T., Willie, J.T., Hara, J., Tsujino, N., Mieda, M., Tominaga, M., Yagami, K., Sugiyama, F., Goto, K., et al. 2003. Hypothalamic orexin neurons regulate arousal according to energy balance in mice. Neuron 38: 701-713. 


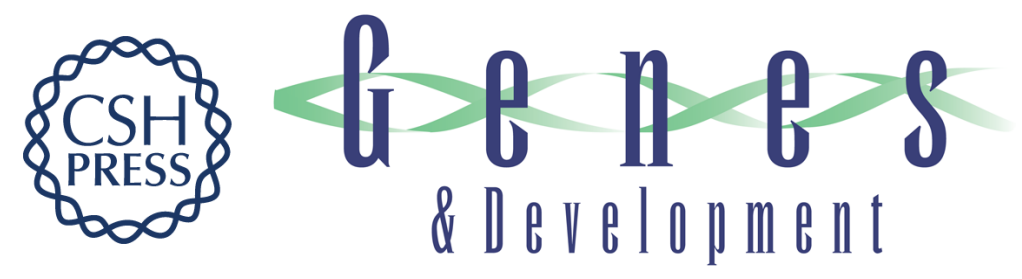

\section{The neurohormone orexin stimulates hypoxia-inducible factor-1 activity}

Devanjan Sikder and Thomas Kodadek

Genes Dev. 2007, 21:

Access the most recent version at doi:10.1101/gad.1584307

Supplemental http://genesdev.cshlp.org/content/suppl/2007/11/01/21.22.2995.DC1
Material

References This article cites 24 articles, 5 of which can be accessed free at:

http://genesdev.cshlp.org/content/21/22/2995.full.html\#ref-list-1

License

Email Alerting

Receive free email alerts when new articles cite this article - sign up in the box at the top

Service

right corner of the article or click here.

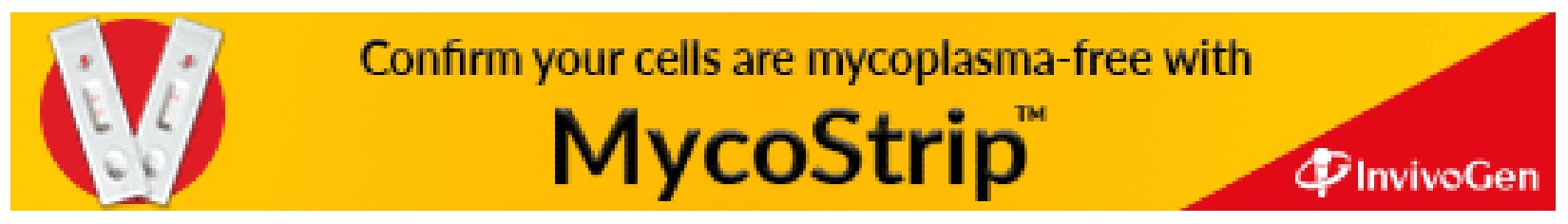

